TUW-96-28

gr-qc/9612012

\title{
Exact Path Integral Quantization of Generic 2-D Dilaton Gravity
}

\author{
W. Kummer ${ }^{1 *}$, H. Liebl ${ }^{1 \dagger}$ and D.V. Vassilevich ${ }^{1,2}$ K $^{\prime}$ \\ ${ }^{1}$ Institut für Theoretische Physik \\ Technische Universität Wien \\ Wiedner Hauptstr. 8-10, A-1040 Wien \\ Austria \\ ${ }^{2}$ Department of Theoretical Physics \\ St. Petersburg University \\ 198904 St. Petersburg \\ Russia
}

\begin{abstract}
Local path integral quantization of generic $2 \mathrm{D}$ dilaton gravity is considered. Locality means that we assume asymptotic fall off conditions for all fields. We demonstrate that in the absence of 'matter' fields to all orders of perturbation theory and for all 2D dilaton theories the quantum effective action coincides with the classical one. This resolves the apparent contradiction between the well established results of Dirac quantization and perturbative (path-integral) approaches which seemed to yield non-trivial quantum corrections. For a particular case, the Jackiw-Teitelboim model, our result is even extended to the situation when a matter field is present.
\end{abstract}

\footnotetext{
*e-mail: wkummer@tph.tuwien.ac.at

$\dagger$ e-mail: liebl@tph16.tuwien.ac.at

$\ddagger$ e-mail: vasilevich@phim.niif.spb.su
} 


\section{Introduction}

Semiclassical calculations, i.e. the quantization of a scalar field on a classical gravitational background are known to lead to fascinating results such as the evaporation of black holes [1]. On the other hand, this additional insight leads to new fundamental problems, e.g. the information paradox or to the question of black hole remnants to name only the most prominent ones. A satisfying answer to the final fate of a singularity can only be extracted from a theory with quantized matter and gravity.

In recent years, stimulated by the 'dilaton black hole' [2, 3] numerous studies of quantized gravity in the simplified setting of $2 \mathrm{D}$ models were performed ([4] to [15]). Louis-Martinez et al. [4] treated generic 2D dilaton gravity in the second order formalism

$$
\left.\mathcal{L}_{(1)}=\sqrt{-g}\left(-X \frac{R}{2}-\frac{U(X)}{2}(\nabla X)^{2}+V(X)\right)\right)
$$

using a Dirac quantization scheme. A gauge theoretical formulation for string inspired gravity was developed and quantized by Cangemi and Jackiw [5]. In ref. [6] their solutions were shown to be equivalent to the ones of [4]. A Dirac approach was recently used to quantize string inspired dilatonic gravity [7]. In an alternative approach spherically symmetric gravity was quantized in Ashtekar's framework by Kastrup [8] and in a geometrodynamical formulation by Kuchař [9]. In particular Strobl [10] has treated a large class of 2D gravity theories within the Poisson-Sigma approach. A common feature of all these studies is that due to the particular structure of the theory the constraints can be solved exactly, yielding a finite dimensional phase space. Then as a consequence of Dirac quantization it is found that quantum effects for only a finite number of variables are observed. Physically this is in agreement with the fact that dilatonic gravity describes no propagating gravitons. Due to the particular structure of the theory the constraints can be solved exactly yielding a finite dimensional reduced phase space. This remarkable property raises hope that in the case of dilatonic gravity one will be able to get insight into the information paradox without being forced to deal with the ultraviolet problems of higher dimensional gravities. Of course, despite of its many appealing features this approach by itself is insufficient to describe Hawking radiation in quantum gravity. In the presence of an additional matter field again an infinite number of modes must be quantized. In order to tackle that problem the results mentioned above first of all should 
be translated into the language of (non perturbative) quantum field theory described by the path integral as the most adequate method for dealing with infinite dimensional quantum systems.

Indeed, one-loop quantum corrections to the classical action and renormalization group equations have been also considered perturbatively 11. Matter fields are easily included in this approach. In this way, however, even pure dilatonic gravity (1) was found to exhibit a highly non-trivial renormalization structure, undermining the main motivation for considering dilatonic gravity as a simple toy model of quantum black hole physics! But even more serious, in our opinion, is the contradiction of these results with the ones from Hamiltonian approaches as described in the last paragraph.

Thus a formalism is required which would combine integrability and simple ultra violet properties of the reduced phase space quantization with the possibility to include matter and obtain the local quantities of the field theoretical quantization.

In the present work we are filling in the gap between the two approaches mentioned above, removing at the same time the apparent contradiction: We demonstrate that in pure dilatonic gravity (1) there are no local quantum corrections in the effective action for the path integral approach as well. To this end we generalize [12, 13] and perform an exact non-perturbative path integral quantization of a generic 2D dilaton model containing all the above models. We give the explicit form of the generating functional for connected Green functions. Adding matter fields in general destroys the functional integrability and suffers therefore from the same weaknesses as the first approach. However, the particular case of JT gravity [14] even in the presence of matter fields allows an exact path integral quantization'.

Main technical features of our approach are the use of the first order action for Cartan variables in the temporal gauge, corresponding to an Eddington Finkelstein (EF) gauge for the metric [12]. Our analysis is local, meaning that we assume asymptotic fall off conditions for all fields. This is enough for the first step of a path integral quantization, which of course, in a second step should be adapted to take into account global effects familiar from the reduced phase space approach.

The paper is organized as follows: In section 2.1 we show the quantum equivalence of the first order formulation of gravity including torsion to generic 2D (torsionless) dilaton gravity. Using the canonical BVF approach

\footnotetext{
${ }^{1}$ For the matterless case this model was quantized exactly already by Henneaux [15].
} 
in section 2.2 we obtain the generating functional and find that there are no quantum corrections to the (matterless) theory. Hence, the effective action coincides with the original classical one and is therefore only calculated as a consistency check. The exact quantization of the JT model including matter is presented in section 3 . Critical remarks on necessary boundary conditions and an outlook on present and future work are contained in section 1 .

\section{Exact Path Integral Quantization}

\subsection{Equivalence of first and second order forms}

Our first goal is to demonstrate the quantum equivalence of the second order form (11) to the first order action

$$
\mathcal{L}_{(2)}=X^{+} D e^{-}+X^{-} D e^{+}+X d \omega+\epsilon\left(V(X)+X^{+} X^{-} U(X)\right),
$$

where $D e^{a}=d e^{a}+(\omega \wedge e)^{a}$ is the torsion two form, the scalar curvature $R$ is related to the spin connection $\omega$ by $-\frac{R}{2}=* d \omega$ and $\epsilon$ denotes the volume two form $\epsilon=\frac{1}{2} \varepsilon_{a b} e^{a} \wedge e^{b}=d^{2} x \operatorname{det} e_{a}^{\mu}=d^{2} x(e)$. Our conventions are determined by $\eta=\operatorname{diag}(1,-1)$ and $\varepsilon^{a b}$ by $\varepsilon^{01}=-\varepsilon^{10}=1$. We also have to stress that even with Greek indices $\varepsilon^{\mu \nu}$ is always understood as the antisymmetric symbol and never as the corresponding tensor. The generating functional for the Green functions is given by

$$
W=\int(\mathcal{D} X)\left(\mathcal{D} X^{+}\right)\left(\mathcal{D} X^{-}\right)\left(\mathcal{D} e_{\mu}^{a}\right)_{g f}\left(\mathcal{D} \omega_{\mu}\right) \exp \left[i \int_{x} \mathcal{L}_{(2)}+\mathcal{L}_{s}\right],
$$

where $\mathcal{L}_{s}$ denotes the Lagrangian containing source terms for the fields. However, since dilaton gravity does not any dependence on $X^{ \pm}$and on $\omega_{\mu}$ we do not introduce the corresponding sources at this point. A suitable gauge fixing is $e_{0}^{-}=e_{1}^{+}=1, e_{0}^{+}=0$. It is easy to check that in the following no division by $e_{0}^{+}$needs to be performed. Also note that $\operatorname{det} g=\operatorname{det} e=1$. Performing

the functional integration with respect to $\omega_{0}$ and $\omega_{1}$ results in

$$
W=\int(\mathcal{D} X)\left(\mathcal{D} X^{+}\right)\left(\mathcal{D} X^{-}\right)\left(\mathcal{D} e_{\mu}^{a}\right) \delta_{\omega_{0}} \delta_{\omega_{1}} \exp \left[i \int_{x} \hat{\mathcal{L}}_{(2)}+\mathcal{L}_{s}\right]
$$

The path integral measure in our gauge is

$$
\left(\mathcal{D} e_{\mu}^{a}\right)_{g f}=F_{F P} \mathcal{D} e_{1}^{-}
$$


where $F_{F P}$ is the Faddeev-Popov factor. We use the abbreviations

$$
\begin{aligned}
\delta_{\omega_{0}} & =\delta\left(\partial_{1} X-X^{+} e_{1}^{-}+X^{-} e_{1}^{+}\right) \\
\delta_{\omega_{1}} & =\delta\left(-\partial_{0} X+X^{+} e_{0}^{-}-X^{-} e_{0}^{+}\right) \\
\hat{\mathcal{L}}_{(2)} & =\varepsilon^{\mu \nu}\left[X^{+} \partial_{\mu} e_{\nu}^{-}+X^{-} \partial_{\mu} e_{\nu}^{+}+e_{\mu}^{+} e_{\nu}^{-}\left(V(X)+X^{+} X^{-} U(X)\right)\right] .
\end{aligned}
$$

Integration over $X^{+}$and $X^{-}$finally yields

$$
W=\int(\mathcal{D} X)\left(\mathcal{D} g_{\mu \nu}\right)_{g f} \exp \left[i \int_{x} \mathcal{L}_{(1)}+\mathcal{L}_{s}\right]
$$

In terms of $g_{\mu \nu}$ our gauge condition becomes the Eddington-Finkelstein gauge $g_{00}=0, g_{01}=1$ with the single unconstrained component $g_{11}=e_{1}^{-}$. The path integral measure becomes

$$
\left(\mathcal{D} g_{\mu \nu}\right)_{g f}=F_{F P} \mathcal{D} g_{11}
$$

with the same Faddeev-Popov determinant as before (5). As a result of the commonly used introduction of that determinant in our gauge $F_{F P}$ even turns out to be field independent. $\mathcal{L}_{(1)}$ is exactly given by (10). To obtain (9) we used $(e) \equiv \sqrt{-g},(e)^{2} g^{\alpha \beta}=\varepsilon^{\alpha \gamma} \varepsilon^{\delta \beta} g_{\gamma \delta}$ and the relation

$$
\tilde{\omega}_{\mu}=\eta_{a b} \frac{\varepsilon^{\alpha \beta}}{(e)} e_{\mu}^{a} \partial_{\alpha} e_{\beta}^{b},
$$

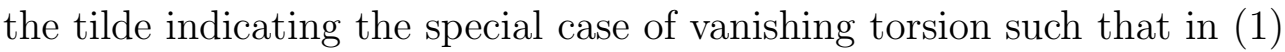

$$
\sqrt{-g} \frac{R}{2}=\varepsilon^{\nu \mu} \varepsilon^{\alpha \beta} \partial_{\mu}\left(\frac{e_{\nu}^{a}}{e} \partial_{\alpha} e_{\beta a}\right) .
$$

Therefore, (11) will only produce the torsionless part of the scalar curvature as it is given in conventional dilaton theories. Of course, an additional conformal transformation of the zweibein would result in additional kinetic terms in the Lagrangian.

Thus the quantum theory of (21) is indeed equivalent to the one from the action (1).

\subsection{Canonical BVF Approach}

As a first step in our quantization program we use 13 the canonical BVF [16] approach in order to obtain the determinants that appear by fixing the 
gauge in (2). We will be working in a 'temporal' gauge which corresponds to an Eddington Finkelstein gauge for the metric defined by:

$$
e_{0}^{+}=\omega_{0}=0 \quad, \quad e_{0}^{-}=1
$$

Let us choose the canonical coordinates to be $q_{i}=\left(\omega_{1}, e_{1}^{-}, e_{1}^{+}\right)$and the corresponding canonical momenta $p^{i}=\left(X, X^{+}, X^{-}\right)$. The Poisson brackets have the form

$$
\left\{q_{i}\left(x^{1}\right), p^{k}\left(y^{1}\right)\right\}=\delta_{i}^{k} \delta, \quad \delta=\delta\left(x^{1}-y^{1}\right)
$$

For the remaining variables $\bar{q}_{i}=\left(\omega_{0}, e_{0}^{-}, e_{0}^{+}\right)$the canonical momenta $\bar{p}_{i}$ vanish (primary constraints). $\bar{q}_{i}$ and $\bar{p}_{i}$ are assumed to obey relations analogous to (14). Dropping a surface term[2, the $\bar{q}^{\prime} s$ can be identified as Lagrange multipliers in the canonical Hamiltonian

$$
\mathcal{H}_{c}=\dot{q}_{i} p_{i}-\mathcal{L}_{(1)}=-\bar{q}_{i} G_{i}
$$

thereby enforcing the secondary constraints

$$
\begin{aligned}
& G_{1}=-X^{+} e_{1}^{-}+X^{-} e_{1}^{+}+\partial_{1} X \\
& G_{2}=\partial_{1} X^{+}+\omega_{1} X^{+}-e_{1}^{+}\left(V(X)+X^{+} X^{-} U(X)\right) \\
& G_{3}=\partial_{1} X^{-}-\omega_{1} X^{-}+e_{1}^{-}\left(V(X)+X^{+} X^{-} U(X)\right) .
\end{aligned}
$$

Their Poisson brackets

$$
\begin{aligned}
\left\{G_{1}, G_{2}\right\}= & -G_{2} \delta \\
\left\{G_{1}, G_{3}\right\}= & G_{3} \delta \\
\left\{G_{2}, G_{3}\right\}= & -\left[\left(V^{\prime}(X)+X^{+} X^{-} U^{\prime}(X)\right) G_{1}+\right. \\
& \left.+X^{-} U(X) G_{2}+X^{+} U(X) G_{3}\right] \delta,
\end{aligned}
$$

show the absence of ternary constraints. The prime denotes differentiation with respect to $X$. It should be noted that (17) is the generalization of 117 where the authors restricted themselves to $R^{2}+T^{2}$ gravity [18. The structure functions $\mathcal{C}_{i j}^{k}$ defined through the equation $\left\{G_{i}, G_{j}\right\}=\mathcal{C}_{i j}^{k} G_{k} \delta$ can be used to construct the BRST charge

$$
\Omega=\int d^{2} x\left(b_{i} \bar{p}^{i}+G_{i} c^{i}+\bar{b}_{k} \mathcal{C}_{i j}^{k} c^{i} c^{j}\right)
$$

\footnotetext{
${ }^{2} \mathrm{~A}$ justification for doing this here and in subsequent steps within our present approach will be given below.
} 
satisfying the nil-potency condition $\{\Omega, \Omega\}=0$. Four types of ghost fields, $b, c, \bar{b}$ and $\bar{c}$ have been introduced, for which the Poisson antibrackets are

$$
\left\{\bar{b}_{i}, c^{j}\right\}=\left\{b_{i}, \bar{c}^{j}\right\}=-\delta_{i}^{j} \delta
$$

Using these ingredients we are able to construct the BRS extension of the canonical Hamiltonian $\mathcal{H}_{\text {ext }}$ and the effective Lagrangian $\mathcal{L}_{\text {eff }}$,

$$
\begin{aligned}
\mathcal{H}_{\mathrm{ext}} & =\mathcal{H}_{c}+c_{i} \bar{q}_{j} \mathcal{C}_{i j}^{k} \bar{b}_{k}-b_{i} \bar{b}_{i} . \\
\mathcal{L}_{\mathrm{eff}} & =\dot{q}_{i} p_{i}+\dot{\bar{q}}_{i} \bar{p}_{i}+\dot{b}_{i} \bar{c}_{i}+\dot{c}_{i} \bar{b}_{i}-\mathcal{H}_{\mathrm{eff}} \\
\mathcal{H}_{\mathrm{eff}} & =\mathcal{H}_{\text {ext }}-\{\Psi, \Omega\},
\end{aligned}
$$

in the sense of BVF. Both $\mathcal{H}_{\text {ext }}$ and $\mathcal{L}_{\text {eff }}$ are BRS invariant:

$$
\left\{\mathcal{L}_{\text {eff }}, \Omega\right\}=\left\{\mathcal{H}_{\text {ext }}, \Omega\right\}=0
$$

In (20) $\Psi$ denotes the gauge fermion which contains the gauge fixing functions $\chi^{i}$ :

$$
\Psi=\bar{c}_{i} \chi_{i}
$$

In the temporal gauge (13), the gauge fixing functions are derived from

$$
\chi_{i}=\frac{1}{\gamma}\left(\bar{q}_{i}-a_{i}\right), \quad a_{1}=a_{3}=0, a_{2}=1
$$

with the limit $\gamma \rightarrow 0$ to be taken later. This yields

$$
\mathcal{H}_{\mathrm{eff}}=\mathcal{H}_{\mathrm{ext}}+\frac{1}{\gamma} \bar{p}_{i}\left(\bar{q}_{i}-a_{i}\right)-\frac{1}{\gamma} \bar{c}_{i} b_{i}
$$

The path integral can be now presented in the form

$$
W=\int(\mathcal{D} q)(\mathcal{D} p)(\mathcal{D} \bar{q})(\mathcal{D} \bar{p})(\mathcal{D} b)(\mathcal{D} \bar{c})(\mathcal{D} \bar{b})(\mathcal{D} c) \exp \left[i \int d^{2} x\left(\mathcal{L}_{e f f}+J p+j q\right)\right]
$$

As a first step the $b$ and $\bar{b}$ integrals are performed. By a change of variables $\bar{p} \rightarrow \gamma \bar{p}$ and $\bar{c} \rightarrow \gamma \bar{c}$ with a unit Jacobian factor and taking the limit $\gamma=0$ we obtain the path integral over the remaining variables with the action

$$
\mathcal{L}^{\prime}=\dot{q}_{i} p_{i}+\bar{q}_{i} G_{i}-\bar{p}_{i}\left(\bar{q}_{i}-a_{i}\right)-c_{i}\left(\delta_{i}^{k} \partial_{0}+\bar{q}_{j} \mathcal{C}_{i j}^{k}\right) \bar{c}_{k}+J p+j q
$$


Integration over $\bar{p}$ and $\bar{q}$ now is equivalent to replacing $\bar{q}_{i}$ by $a_{i}$ everywhere in (26) as it should be according to (13). Finally integration over $c$ and $\bar{c}$ yields the determinant

$$
F=\operatorname{det}\left(\delta_{i}^{k} \partial_{0}+\mathcal{C}_{i 2}^{k}\right)=\left(\operatorname{det} \partial_{0}\right)^{2} \operatorname{det}\left(\partial_{0}+X^{+} U(X)\right) .
$$

$F$ will be cancelled by the determinants produced by integrating over $X^{ \pm}$ and $X$ below. Note that the operator $\left(\partial_{0}+X^{+} U(X)\right)$ is unitary equivalent to $\partial_{0}:\left(\partial_{0}+X^{+} U(X)\right)=e^{-y} \partial_{0} e^{y}, \partial_{0} y=X^{+} U(X)$. Hence $\operatorname{det}\left(\partial_{0}+X^{+} U(X)\right)$ is independent of $X^{+} U(X)$.

After having determined the contributions of the gauge fixing we will now integrate over the remaining fields. For this reason we introduce sources $\left(j^{ \pm}, j, J^{ \pm}, J\right)$ for the fields $\left(e_{1}^{\mp}, \omega_{1}, X^{\mp}, X\right)$, respectively.

The generating functional for the Green functions is

$$
W=\int(\mathcal{D} X)\left(\mathcal{D} X^{+}\right)\left(\mathcal{D} X^{-}\right)\left(\mathcal{D} e_{1}^{+}\right)\left(\mathcal{D} e_{1}^{-}\right)\left(\mathcal{D} \omega_{1}\right) F \exp \left[i \int_{x} \mathcal{L}_{(2)}+\mathcal{L}_{s}\right]
$$

where $F$ denotes the determinant from the previous section, $\mathcal{L}_{(2)}$ is the gauge fixed part of the Lagrangian (2) and $\mathcal{L}_{s}$ denotes the contribution of the sources:

$$
\mathcal{L}_{s}=j^{+} e_{1}^{-}+j^{-} e_{1}^{+}+j \omega_{1}+J^{+} X^{-}+J^{-} X^{+}+J X
$$

Integrating over the zweibein components and the spin connection results in

$$
W=\int(\mathcal{D} X)\left(\mathcal{D} X^{+}\right)\left(\mathcal{D} X^{-}\right) \delta_{(1)} \delta_{(2)} \delta_{(3)} F \exp \left[i \int J^{+} X^{-}+J^{-} X^{+}+J X\right]
$$

with

$$
\begin{aligned}
& \delta_{(1)}=\delta\left(-\partial_{0} X^{+}+j^{+}\right) \\
& \delta_{(2)}=\delta\left(-\left(X^{+} U(X)+\partial_{0}\right) X^{-}+j^{-}-V(X)\right) \\
& \delta_{(3)}=\delta\left(-\partial_{0} X+X^{+}+j\right) .
\end{aligned}
$$

We must stress again that due to the partial integrations where we discarded the surface terms the following results are true only locally with a proper definition of what we mean when Green functions like $\partial_{0}^{-1}, \partial_{0}^{-2}$ appear. 
The remaining integrations can be performed most conveniently in the order $X^{+}, X^{-}$and finally $X$ to yield for the generating functional of connected Green functions

$$
Z=-i \ln W=\int J X+J^{-} \frac{1}{\partial_{0}} j^{+}+J^{+} \frac{1}{\partial_{0}+U(X) \frac{1}{\partial_{0}} j^{+}}\left(j^{-}-V(X)\right)
$$

where $X$ has to be replaced by

$$
X=\frac{1}{\partial_{0}^{2}} j^{+}+\frac{1}{\partial_{0}} j
$$

It should be stressed that the determinant $F$ is precisely canceled by the determinants appearing during these last three integrations. Eq.(34) gives the exact non-perturbative generating functional for connected Green functions and it does not contain any divergences, because it clearly describes tree-graphs only. Hence no quantum effects remain.

Now we turn to the ill defined expressions $\left(\partial_{0}\right)^{-1}$. As shown in [12] a proper (vanishing) asymptotic behavior results from a regularization

$$
\begin{aligned}
\frac{1}{\partial_{0}{ }^{2}} & =\lim _{\mu^{2} \rightarrow 0} \frac{1}{\partial_{0}^{2}+\mu^{2}} \\
\frac{1}{\partial_{0}} & =\partial_{0}\left(\frac{1}{\partial_{0}^{2}}\right)
\end{aligned}
$$

where $\mu^{2}$ insures a proper infrared cutoff. Since each partial integration above involved either $X, X^{+}$or $X^{-}$which in turn all exhibit at least a $\partial_{0}^{-1}$ behavior, all our previous steps are justified.

\subsection{Effective Action $\Gamma$}

It is illustrative to calculate the effective action $\Gamma$ which generates one particle irreducible vertex functions. $\Gamma$ is defined through a Legendre transformation of the generating functional for connected Green functions

$\Gamma\left(\bar{X}^{ \pm}, \bar{X}, \bar{e}_{1}^{ \pm}, \bar{\omega}_{1}\right)=Z(J, j)-\int\left(J^{+} \bar{X}^{-}+J^{-} \bar{X}^{+}+J \bar{X}+j \bar{\omega}_{1}+j^{+} \bar{e}_{1}^{-}+j^{-} \bar{e}_{1}^{+}\right)$.

Here $\bar{X}, \bar{e}, \bar{\omega}$ denote the corresponding 'mean fields' in the presence of sources, e.g.

$$
\bar{X}^{ \pm}=\frac{\delta Z}{\delta J^{\mp}}
$$


In the following we drop the bars because no confusion can arise with the variables in the previous subsection. Only three such equations are needed:

$$
\begin{aligned}
X & =\frac{1}{\partial_{0}}\left(\frac{1}{\partial_{0}} j^{+}+j\right) \\
X^{+} & =\frac{1}{\partial_{0}} j^{+} \\
X^{-} & =\frac{1}{\partial_{0}+U(X) X^{+}}\left(j^{-}-V(X)\right)
\end{aligned}
$$

By substituting (40) in (38) we obtain

$$
\Gamma=\int\left(-e_{1}^{+}\left(\partial_{0}+U(X) X^{+}\right) X^{-}-e_{1}^{+} V(X)-e_{1}^{-} \partial_{0} X^{+}-\omega_{1} \partial_{0} X+\omega_{1} X^{+}\right)
$$

Up to surface terms, this is exactly the classical action (2) in the temporal gauge (13). As demonstrated already above for the connected Green functions, there are no quantum corrections to the theory, (41) is certainly the expected result and can hence be considered as a consistency check.

Our result means that up to possible global effects the quantum effective action should coincide with the classical one on shell in any parameterization for either the first or second order formalism. Of course, in a framework of perturbative expansions for certain parameterizations in configuration space, combined with unsuitable gauge fixings in the case of gauge theories, artificial divergences can appear, a familiar example being the non-renormalizable ones of individual graphs in the unitary gauge of Yang Mills theories. In dimensional regularization with the background field gauge in [11] divergent counterterms were produced which are indeed pure artifacts of this approach. The essential indication for that is their vanishing on shell. To illustrate this point let us consider a generic theory described by a classical action $S(\Phi)$. The one-loop effective action is given by

$$
Z_{\Phi}^{1-\text { loop }} \propto \log \operatorname{det}\left(\frac{\delta S}{\delta \Phi^{A} \delta \Phi^{B}}\right)
$$


In another parameterization $\phi(\Phi)$, we have

$$
\begin{array}{r}
Z_{\phi}^{1-\text { loop }} \propto \log \operatorname{det}\left(\frac{\delta S}{\delta \phi^{a} \delta \phi^{b}}\right) \\
=\log \operatorname{det}\left(\frac{\delta S}{\delta \Phi^{A} \delta \Phi^{B}} \frac{\delta \Phi^{B}}{\delta \phi^{b}} \frac{\delta \Phi^{A}}{\delta \phi^{a}}+\frac{\delta S}{\delta \Phi^{C}} \frac{\delta^{2} \Phi^{c}}{\delta \phi^{a} \delta \phi^{b}}\right)
\end{array}
$$

Since the factors $\delta \Phi / \delta \phi$ can be absorbed into a redefined path integral measure, on shell (42) coincides with (43). This is not true off shell. Even if (42) is finite, (43) may contain divergences. Our result proves that the latter may be cancelled by a suitable all-order counterterm as in [12] for all $2 \mathrm{D}$ dilaton theories.

\section{$3 \quad$ JT Model with Matter}

Up to now we have quantized theories of pure gravity ignoring the effects of any matter contribution. In this section we shall minimally couple a scalar field to our gravitational action. The standard procedure for path integral quantization of the scalar field (in the absence of sources for that field) amounts to determine

$$
\operatorname{det} \square=\operatorname{det} \frac{1}{\sqrt{-g}} \partial^{\mu} \sqrt{-g} g_{\mu \nu} \partial^{\nu}
$$

which in turn (see e.g. [19]) generates the effective nonlocal action

$$
\mathcal{L}_{P}=\sqrt{-g} R \frac{1}{\square} R
$$

also called Polyakov action. $R$ denotes the scalar curvature for vanishing torsion (12), which in our temporal gauge (13) becomes

$$
R=\frac{2}{e_{1}^{+}}\left(\partial_{0} \partial_{0} e_{1}^{-}+\partial_{0}\left(\frac{e_{1}^{-}}{e_{1}^{+}} \partial_{0} e_{1}^{+}\right)-\partial_{1}\left(\frac{1}{e_{1}^{+}} \partial_{0} e_{1}^{+}\right)\right) .
$$

The d'Alembertian as defined in (44) has to be expressed in terms of the zweibeins which in our gauge leads to

$$
\square=\frac{1}{e_{1}^{+}}\left(\left(\partial_{1}-e_{1}^{-} \partial_{0}\right) e_{1}^{+} \partial_{0}+\partial_{0} e_{1}^{+} \partial_{1}-e_{1}^{-} \partial_{0} e_{1}^{+} \partial_{0}\right) .
$$

\footnotetext{
${ }^{3}$ corresponding to a canonical transformation in phase space
} 
We see that (45) is not linear in the zweibein anymore. Therefore, in general, adding the Polyakov term to our first order action (2) will destroy the procedure of section 2. However, there exists a particular case, the wellknown Jackiw Teitelboim (JT) model [14] for which we are able to perform a complete integration of the gravitational and the matter action. The JT model is obtained by setting $U(X)=0$ and $V(X)=\alpha X$, with $\alpha$ being a constant. Performing the integration, all the steps up to (28) and (29) remain the same because the Polyakov term is not affected. Due to the complicated dependence of this term on the zweibein one cannot analytically integrate these fields. Instead we now perform the $X, X^{+}$and $X^{-}$integration first to arrive (starting from (28)) at

$$
W=\int\left(\mathcal{D} e_{1}^{+}\right)\left(\mathcal{D} e_{1}^{-}\right)\left(\mathcal{D} \omega_{1}\right) \delta_{(X)} \delta_{\left(X^{+}\right)} \delta_{\left(X^{-}\right)} F \exp i \int \mathcal{L}_{e f f}
$$

with

$$
\begin{aligned}
\mathcal{L}_{e f f} & =j \omega_{1}+j^{+} e_{1}^{-}+j^{-} e_{1}^{+}+\mathcal{L}_{P} \\
\delta_{(X)} & =\delta\left(\partial_{0} \omega_{1}+J-\alpha e_{1}^{+}\right) \\
\delta_{\left(X^{+}\right)} & =\delta\left(\omega_{1}+J^{-}+\partial_{0} e_{1}^{-}\right) \\
\delta_{\left(X^{-}\right)} & =\delta\left(\partial_{0} e_{1}^{+}+J^{+}\right)
\end{aligned}
$$

in a suggestive notation. The remaining integrations can be done with the use of (50) to (52). A factor of $\left(\operatorname{det} \partial_{0}\right)^{-3}$ appearing during these calculations again is cancelled by the term $F$ under the integral. As a final result we arrive at

$$
Z=-i \ln W=\int \mathcal{L}_{e f f}
$$

$\mathcal{L}_{\text {eff }}$ being defined by (49). The zweibein and connection are understood to be expressed as

$$
\begin{aligned}
e_{1}^{-} & =\frac{1}{\left(\partial_{0}\right)^{3}}\left(\partial_{0} J+\alpha J^{+}-\partial_{0} \partial_{0} J^{-}\right) \\
e_{1}^{+} & =-\frac{1}{\partial_{0}} J^{+} \\
\omega_{1} & =-\frac{1}{\partial_{0}} J-\frac{\alpha}{\left(\partial_{0}\right)^{2}} J^{+} .
\end{aligned}
$$


Thus in (53) we succeeded to give the exact non-perturbative generating functional for connected Green functions, quantized 'locally', even in the presence of matter. Our result means that in the absence of external matter sources the quantum JT model with quantum matter is locally equivalent to the classical JT model with the Polyakov term, i.e. the 'semiclassical' approximation becomes exact. It has to be stressed that with the above method the JT model can be treated with any kind of matter (also including fermions 20 ) as long as there is no coupling to $X$ or $X^{ \pm}$. This is clear from the fact that the path integral quantization of the matter field results in a purely geometrical term and the subsequent integrations of the zweibein and connection can always be achieved with the help of (50) to (52).

\section{Remarks and Outlook}

We conclude that the path integral approach is very efficient for the quantization of dilatonic gravity. We were able to quantize the wide class of models (1). This is the counterpart of the reduced phase space quantization as considered by Louis-Martinez et al [4]. However, it must be noted that they actually quantized a model whose kinetic term for the dilaton field was removed by means of a conformal transformation. However, as shown in detail in 21 such transformations drastically change the global structure of the theory already at the classical level, and it is therefore by no means clear how the quantum theory is affected. As we demonstrated here, local quantum effects are not affected by that conformal transformation. There are sources of conformal non-invariance (see e.g. [22, 23]) which may change global effects. Therefore this problem deserves further investigation.

Although we succeeded to quantize 'all' 2D dilaton gravity models in a (non-perturbative) path integral formulation and to remove in this way the existing apparent contradiction to previous Hamiltonian approaches, some self-critical remarks are in order. We have systematically discarded surface integrals produced by partial integrations. This approach was implemented by applying an infrared cutoff. However, since the zweibein will rather exhibit a certain nonvanishing behavior at 'infinity' (e.g. $e_{1}^{+} \rightarrow 1$ for flat space-time) than go to zero, this procedure may be questionable. The nontrivial quantum effects from nontrivial global compactifications including kink solutions [24], now well known for the Hamiltonian approach, are eliminated under

our assumptions. A more elaborate treatment, namely keeping track of all 
boundary terms and the application of proper, preferably gauge independent boundary conditions [25] is currently under progress [26]. This - in our opinion - would provide the only way for a proper path integral treatment, including global effects as well.

\section{Acknowledgement}

This work has been supported by Fonds zur Förderung der wissenschaftlichen Forschung (FWF) Project No. P 10221-PHY. One of the authors (D.V.) thanks GRACENAS for financial support.

\section{References}

[1] S.W. Hawking, Nature (London) 248, (1974), 30; Commun. Math. Phys. 43, (1975), 199

[2] G. Mandal, A. Sengupta and S.R. Wadia, Mod. Phys. Lett. A6, (1991), 1685 (preprint IASSNS-HEP-91/10); E. Witten, Phys. Rev. D44, (1991), 314; V. P. Frolov, Phys. Rev. D46, (1992), 5383; J. G. Russo and A. A. Tseytlin, Nucl. Phys. B382, (1992), 259; J. Russo, L. Susskind, and L. Thorlacius, Phys. Lett. B292, (1992), 13; T. Banks, A. Dabholkar, M. Douglas, and M. O'Laughlin, Phys. Rev. D45, (1992), 3607; S. P. deAlwis, Phys. Lett. B289, (1992), 278;

[3] D. Louis-Martinez, J. Gegenberg and G. Kunstatter, Phys. Lett. B321, 193 (1994); Phys. Rev. D51, (1995), 1781; W.M. Seiler and R.W. Tucker, Phys. Rev. D53, (1996), 4366; A. Barvinsky and G. Kunstat-

ter, Exact Physical Black Hole States in Generic 2-D Dilaton Gravity hep-th/9606134

[4] D. Louis-Martinez, J. Gegenberg and G. Kunstatter, Phys. Lett. B321, (1994), 193

[5] D. Cangemi and R. Jackiw, Phys. Rev. D50, (1994), 3913; Phys. Lett. B337, (1994), 271

[6] E. Benedict, Phys. Lett. B340, (1994), 43 
[7] K.V. Kuchař, J.D. Romano and M. Varadarajan, Dirac constraint quantization of a dilatonic model of gravitational collapse, hep-th/9608011, tentatively scheduled for Phys. Rev. D54, (1996), 12

[8] T. Thiemann and H.A. Kastrup, Nucl. Phys. B399, (1993), 211; H.A. Kastrup and T. Thiemann, Nucl. Phys. B425, (1994), 665

[9] K. Kuchař, Phys. Rev. D50, (1994), 3961

[10] T. Strobl, Phys. Rev. D50, (1994), 7346

[11] J.G. Russo, A.A. Tseytlin, Nucl. Phys. B382, (1992), 259; R. Kantowski and C. Marzban, Phys. Rev. D46, (1992), 5449; E. Elizalde, S. Naftulin and S.D. Odintsov Int. J. Mod. Phys. A9, (1994), 933; E. Elizalde, P. Fosalba-Vela, S. Naftulin and S. D. Odintsov, Phys. Lett. B352, (1995), 235

[12] W. Kummer and D.J. Schwarz, Nucl. Phys. B382, (1992), 171

[13] F. Haider and W. Kummer, Int. J. Mod. Phys. A9, (1994), 207

[14] C. Teitelboim, Phys.Lett. 126B, (1983), 41; R. Jackiw, 1984 Quantum Theory of Gravity, ed S. Christensen (Bristol: Hilger) p.403

[15] M. Henneaux, Phys. Rev. Lett. 54, (1985), 959

[16] E.S. Fradkin and G.A. Vilkovisky, Phys. Lett. B55, (1975), 244; I.A. Batalin and G.A. Vilkovisky, Phys. Lett. B69, (1977), 309; E.S. Fradkin and T.E. Fradkina, Phys. Lett. B72, (1978), 343

[17] H. Grosse, W. Kummer, P. Prešnajder and D.J. Schwarz, J. Math. Phys. 33 (11) (1992), 3892

[18] M.O. Katanaev and I.O. Volovich, Phys. Lett. 175B, (1986), 413

[19] E. Abdalla, M.C.B. Abdalla and K.D. Rothe, Non-Perturbative Methods in Two Dimensional Quantum Field Theory, World Scientific, (1991), p. 605

[20] W. Kummer, Exact Classical and Quantum Integrability of $R^{2}+T^{2}$ Gravity in $1+1$ Dimensions, Proc. Int. Europhys. Conf. on High Energy Physics, (Eds. J. Carr and M. Perrottet), Edition Frontieres, Gif-surYvette, (1994), 251 - 253 
[21] M.O. Katanaev, W. Kummer and H. Liebl, Phys. Rev. D53, (1996), 5609 and 'On the Completeness of the Black Hole Singularity in $2 d$ Dilaton Theories', gr-qc/9602040, to be published in Nucl. Phys. B

[22] K.C.K. Chan, J.D.E. Creighton and R.B. Mann, Phys. Rev. D54, (1996), 3892.

[23] H.Liebl, D. Vassilevich and S. Alexandrov, Hawking radiation and masses in generalized dilaton theories, gr-qc/9605044

[24] P. Schaller and T. Strobl, Class. Quant. Grav. 11, (1993), 331; T. Strobl, Phys. Rev. D50, (1994), 7356; P. Schaller and T. Strobl, Mod. Phys. Lett. A9, (1994), 3129.

[25] D.V. Vassilevich, Mod. Phys. Lett. A10, (1995), 2239

[26] W. Kummer, H. Liebl and D.V. Vassilevich, in preparation 